NOUVELLE

\section{Le devenir des cellules SDF}

Olivier Meilhac, Jean-Baptiste Michel
Inserm U.460,

CHU Xavier Bichat, secteur Claude Bernard,

46, rue Henri Huchard, 75877 Paris Cedex 18, France. meilhac@bichat.inserm.fr
> L'anoïkis, du grec ancien «sans domicile fixe », représente un mode d'élimination physiologique des cellules par un mécanisme d'apoptose consécutif à une rupture des communications entre une cellule $(\rightarrow) \mathrm{m} / \mathrm{s}$ 2005, $n^{\circ} 2$, p. 117 et son environnement $(\rightarrow)$. D'abord décrite pour des cellules épithéliales $[1,2]$, cette apoptose par détachement a été identifiée depuis dans d'autres types cellulaires, épithéliaux ou mésenchymateux. Alors qu'un excès de mort par anoïkis observé dans un contexte protéolytique (dans lequel les communications cellules-ma-trice extracellulaire sont rompues) participe aux maladies dégénératives, à l'inverse, une résistance marquée au phénomène d'anoïkis permet aux cellules cancéreuses de se disséminer.

\section{Signaux de survie}

Les communications entre la matrice extracellulaire et les cellules s'effectuent essentiellement via les intégrines, hétérodimères qui, selon les isoformes qui les composent, ont des affinités plus ou moins prononcées pour la fibronectine, la vitronectine, le collagène... Cette liaison entre les intégrines et les protéines de la matrice extracellulaire est à l'origine d'une cascade de signaux inhibant l'apoptose. Le domaine intracellulaire des intégrines (notamment la sous-unité $\beta$ ) recrute des protéines se liant au réseau d'actine, ainsi que des protéines de signalisation comme FAK (focal adhesion kinase) et ILK (integrin linked kinase). L'activation de la sérine-thréonine kinase Akt est à l'origine de signaux antiapoptotiques conduisant à l'inhibition de la transcription de gènes pro-apoptotiques et à l'activation de celle de gènes antiapoptotiques. De plus, Akt, en phosphorylant $\mathrm{Bad}$, inhibe sa dimérisaton avec $\mathrm{Bcl}-2$, qui peut jouer son rôle anti-apoptotique au niveau mitochondrial (pour revue, voir [3]). L'absence de signaux de survie, comme dans le cas d'une rupture des liaisons matrice extracellulaire-intégrines, débride la transcription de gènes proapoptotiques et inhibe la fonction de protéines anti-apoptotiques comme Bcl-2, qui s'héterodimérise avec Bad. La «tenségrité» (de l'anglais tensional integrity) correspond aux forces de tension produites par les communications entre le cytosquelette et l'environnement cellulaire (jonctions intercellulaires ou cellule-matrice); elle participe à l'envoi de signaux de survie, puisque, lorsque les microfilaments d'actine et les microtubules sont dissociés, un programme d'apoptose impliquant des voies similaires à l'anoïkis est mis en route [4]. Enfin, les communications intercellulaires impliquant les cadhérines dans les tissus semblent également être importantes dans l'envoi de signaux de survie. Les cadhérines- $\varepsilon$ pourraient, par l'activation du récepteur de l'epidermal growth factor (દGF), augmenter l'expression de $\mathrm{Bcl}-2$ [5]. La perte de telles interactions participerait au déclenchement du phénomène d'anoïkis (Figure 1).

\section{Médiateurs de l'anoïkis}

Si beaucoup de travaux portent sur la signalisation intracellulaire associée au processus d'anoïkis, peu d'études s'intéressent aux communications extracellu- laires du détachement. Même dans les situations d'anoïkis physiologique comme le renouvellement d'un épithélium, les événements déclencheurs du détachement de la cellule de son support sont mal connus. Différents médiateurs extracellulaires de l'anoïkis ont été proposés dans les maladies cardiovasculaires où l'apoptose est impliquée, comme la désendothélialisation, l'insuffisance cardiaque, la fragilisation de la plaque d'athérome et la disparition des cellules musculaires lisses dans les parois anévrismales [6]. L'excès d'activité protéase joue un rôle majeur dans la rupture des communications entre cellules et matrice péricellulaire ; ces protéases peuvent être d'origine inflammatoire (élastase, cathepsines, chymase, tryptase, granzymes) ou résulter de l'activation de zymogènes (activation du plasminogène, de la prothrombine...) [7-9]. Ces phénomènes d'activation tissulaire sont en majeure partie dus à la liaison de ces enzymes à des structures macromoléculaires (gel de fibrine, membranes cellulaires, substances amyloïdes), liaisons qui les rendent en partie inaccessibles à leurs inhibiteurs naturels circulants.

\section{Résistance à l'anoïkis}

Alors qu'un excès d'anoïkis mène à des situations pathologiques dégénératives, avec une raréfaction cellulaire et une accumulation de matrice de remplacement, la survie des cellules détachées de leur support et leur capacité à proliférer pourrait être à l'origine d'un phénomène de dissémination. La survie des cellules endométriales présentes dans la cavité périto- 
néale au cours de rétromenstruations pourrait être un élément-clé dans le développement de l'endométriose [10].

De plus, tous les éléments qui favorisent la survie des cellules détachées et leur adhérence sur un nouveau substrat pourraient être propices à la dissémination et à la prolifération des cellules cancéreuses. $S$. Douma et al. [11] ont récemment montré que des cellules épithéliales surexprimant le récepteur TrkB (dont le ligand principal est le bone derived neurotrophic factor, BDNF) sont résistantes à l'anoïkis. Ce récepteur à activité tyrosine-kinase active la voie de survie phosphatidylinositol 3kinase/Akt. Le BDNF est notamment produit par l'endothélium en conditions d'hypoxie. Ces auteurs ont montré que des cellules normalement adhérentes survivent en suspension lorsqu'elles expriment TrkB, se multiplient et sont capables de provoquer des tumeurs quand elles sont injectées à des souris. La survie de cellules détachées de la matrice extracellulaire pourrait aussi être assurée par les relations intercellulaires impliquant les cadhérines; cette survie prolongée de groupes de cellules détachées, par rapport à une seule cellule détachée de son substrat, a récemment été nommée synoïkis [5].

Enfin, au cours de la mitose, des cellules peuvent se détacher de la matrice et participer au phénomène de métastase. J.M. Vasiliev et al. [12] ont montré que l'activation des petites GTPases de la famille Rho provoquent un état de contraction cellulaire favorisant une réorientation du fuseau mitotique. La cytocinèse se faisant parallèlement au plan d'adhérence cellulaire, la cellule fille pourrait se détacher pour réadhérer et se multiplier loin de son site d'origine. Ainsi, l'activation excessive de RhoA favoriserait par ce mécanisme la dissémination de cellules capables de proliférer, comme c'est le cas au cours du processus de métastase. Le devenir de ces cellules nomades est très dépendant de leur capacité à survivre pendant un temps donné (capacité de résistance à l'anoïkis), puis éventuellement à ré-adhérer et à proliférer. L'homéostasie cellulaire est un équilibre précaire qui participe en permanence au modelage phy- siologique des tissus. L'anoïkis - ou mort cellulaire secondaire à la perte d'interactions entre la cellule et son environnement - est un des mécanismes de cette homéostasie. Son dérèglement participe des phénomènes pathologiques touchant les tissus: résistance à l'anoïkis dans le cancer, excès d'anoïkis dans les maladies dégénératives. L'importance de ce phénomène fait de ses effecteurs moléculaires extra- et intracellulaire des cibles thérapeutiques d'intérêt. $\diamond$

The fate of homeless cells

\section{RÉFÉRENCES}

1. Meredith JE Jr, Fazeli B, Schwartz MA. The extracellular matrix as a cell survival factor. Mol Biol Cell 1993 ; $4: 953-61$.

2. Frisch SM, Francis H. Disruption of epithelial cellmatrix interactions induces apoptosis. J Cell Biol $1994 ; 124: 619-26$.

3. Downward J. PI 3-kinase, Akt and cell survival. Semin Cell Dev Biol 2004 ; 15 : 177-82.

4. Flusberg DA, Numaguchi $Y$, Ingber DE. Cooperative control of Akt phosphorylation, bcl-2 expression, and apoptosis by cytoskeletal microfilaments and microtubules in capillary endothelial cells. Mol Biol Cell $2001 ; 12$ : 3087-94.

5. Shen X, Kramer RH. Adhesion-mediated squamous cell carcinoma survival through ligand-independent activation of epidermal growth factor receptor. Am J Pathol 2004 ; 165 : 1315-29.

6. Michel JB. Anoikis in the cardiovascular system: Known and unknown extracellular mediators. Arterioscler Thromb Vasc Biol 2003 ; 23 : 2146-54.

7. Meilhac 0 , Ho-Tin-Noe B, Houard X, et al. Pericellular plasmin induces smooth muscle cell anoikis. FASEB J 2003; 17 : 1301-3.

8. Rossignol P, Bouton MC, Jandrot-Perrus M, et al. A paradoxical pro-apoptotic effect of thrombin on smooth muscle cells. Exp Cell Res 2004 ; 299 : 279-85.

9. Rossignol P, Ho-Tin-Noe B, Vranckx R, et al. Protease nexin- 1 inhibits plasminogen activation-induced apoptosis of adherent cells. J Biol Chem 2004 ; 279 : 10346-56.

10. Harada T, Kaponis A, Iwabe T, et al. Apoptosis in human endometrium and endometriosis. Hum Reprod Update $2004 ; 10$ : 29-38.

11. Douma S, Van Laar T, Zevenhoven J, et al. Suppression of anoikis and induction of metastasis by the neurotrophic receptor TrkB. Nature 2004 ; $430: 1034-9$.

12. Vasiliev JM, Omelchenko T, Gelfand IM, et al. Rho overexpression leads to mitosis-associated detachment of cells from epithelial sheets: a link to the mechanism of cancer dissemination. Proc Natl Acad Sci USA 2004 ; 101 : 12526-30.

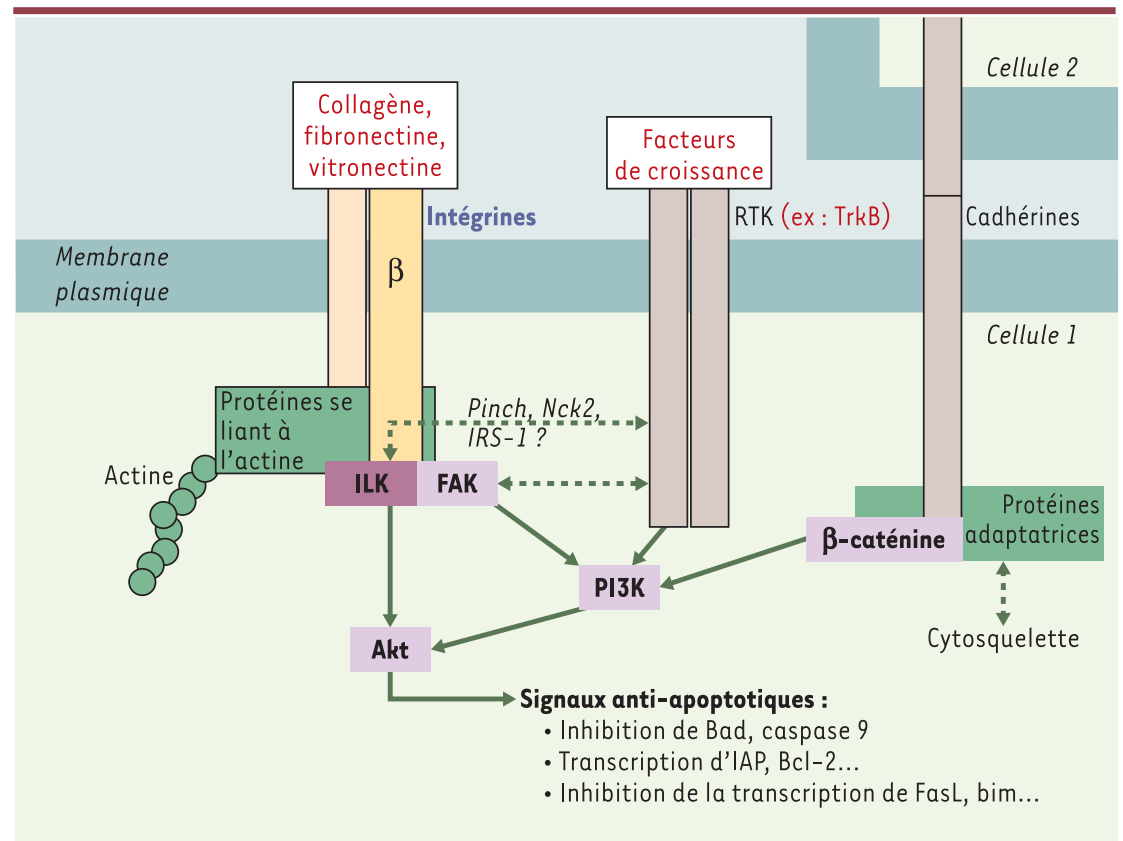

Figure 1. Coopération entre les intégrines, les cadhérines et les récepteurs à activité tyrosinekinase (RTK) pour l'envoi de signaux de survie via Akt. Les communications cellule-matrice et cellule-cellule sont responsables du maintien de la «tenségrité» via des interactions avec les protéines du cytosquelette. Les signaux de survie qu'elles véhiculent passent en partie par Akt, une sérine-thréonine kinase qui active des voies anti-apoptotiques. PINCH : particularly interesting new cystein-histidine-rich protein; FAK : focal adhesion kinase; ILK : integrin-linked kinase; IRS-1 : insulin receptor substrate-1; PI3K: phosphatidylinositol 3-kinase; IAP: inhibitor of apoptosis protein; FasL: ligand de Fas. 